\title{
Monitoring Heat Treatments in Steels by a Non Destructive Ultrasonic Method
}

\author{
Linton Carvajal ${ }^{*}$, Alfredo Artigas ${ }^{a}$, Alberto Monsalve $^{a}$, Elizabeth Arévalo $^{a}$ \\ ${ }^{a}$ Department of Metallurgical Engineering, Universidad de Santiago de Chile, Av. Libertador Bernardo \\ O'Higgins 3363, Estación Central, Santiago, Chile.
}

Received: December 13, 2016; Revised: July 25, 2017; Accepted: July 27, 2017

\begin{abstract}
In order to determine if heat treatments can be discerned by nondestructive ultrasonic testing, samples of SAE 1045 and SAE 4140 steels were subjected to the classical heat treatments of annealing, normalizing, quenching, and quenching and tempering, and their elastics constants, Young's, shear and Poisson's moduli, were monitored by ultrasound. Results show that the microstructural differences associated to the various heat treatments generate differences on the elastic constants that can be effectively discerned by the use of longitudinal and shear ultrasonic waves. Special attention is given to Poisson's modulus, since for its determination only times of flight of longitudinal and shear ultrasonic waves, and not the distance the waves travel, are required, which is of quite practical importance, since measurements are simplified and a fundamental source of error is eliminated. It is thus shown that the microstructural evolution of a steel part subjected to heat treatments can be monitored by this simple ultrasonic method.
\end{abstract}

Keywords: Ultrasound, Heat Treatment, Elastic Constants.

\section{Introduction}

Based on the relation between the phase velocity of sound waves and second order elastic constants, ultrasound is commonly used to determine these parameters in different materials. Though in principle the related equations were developed considering a homogeneous medium ${ }^{1}$, they can be successfully used to determine the effective, or average, constants of a heterogeneous multiphase or polycrystalline material. Most common engineering materials are either slightly othotropic, such as rolled plates, or nearly isotropic. In the former case, knowledge of twelve elastic constants is required for complete characterization; however, only nine are independent, these being: $\mathrm{C}_{11}, \mathrm{C}_{22}, \mathrm{C}_{33}, \mathrm{C}_{44}, \mathrm{C}_{55}, \mathrm{C}_{66} ; \mathrm{C}_{12}$, $\mathrm{C}_{13}$ and $\mathrm{C}_{23}$. In the latter case, $\mathrm{C}_{11}=\mathrm{C}_{22}=\mathrm{C}_{33}$, and $\mathrm{C}_{44}=\mathrm{C}_{55}=$ $\mathrm{C}_{66}=1 / 2\left(\mathrm{C}_{11}-\mathrm{C}_{12}\right)$, so an isotropic material exhibits only three constants, two of which are independent ${ }^{2}$. The following relations allow obtaining them by simply measuring the velocities of two ultrasonic waves with mutually perpendicular polarization directions (i.e., of displacement of the medium's particles), propagating in any direction through the bulk of the material ${ }^{1}$ :

$$
\mathrm{C}_{11}=\rho \mathrm{v}_{i i}^{2} \equiv \rho \mathrm{v}_{\mathrm{L}}^{2} ; \mathrm{C}_{44}=\rho \mathrm{v}_{i j}^{2} \equiv \rho \mathrm{v}_{\mathrm{T}}^{2} ; \mathrm{C}_{12}=\rho\left(\mathrm{v}_{\mathrm{L}}^{2}-2 \mathrm{v}_{\mathrm{T}}^{2}\right) ;
$$

where $\rho$ is the density of the medium, $\mathrm{v}_{i i} \equiv \mathrm{v}_{\mathrm{T}}$ is the velocity of a longitudinal wave (longitudinally polarized to its direction of propagation $i$ ) and $\mathrm{v}_{\mathrm{ij}} \equiv \mathrm{v}_{\mathrm{T}}$, with $i \neq j$, is the velocity of a shear wave (polarized in the direction $j$, transverse to its direction of propagation $i$ ).

* e-mail: linton.carvajal@usach.cl
For the isotropic case, the theory of elasticity ${ }^{2}$ relates the elastic stiffness constants with Young's modulus E, shear modulus G, and Poisson's ratio $v$. Hence, these parameters may be expressed in terms of the velocities of the longitudinal and shear waves ${ }^{3}$ :

$$
\begin{gathered}
E=\frac{\rho \mathrm{v}_{\mathrm{T}}^{2}\left[3\left(\mathrm{v}_{\mathrm{L}} / \mathrm{v}_{\mathrm{T}}\right)^{2}\right]-4}{\left(\mathrm{v}_{\mathrm{L}} / \mathrm{v}_{\mathrm{T}}\right)^{2}-1} \\
\mathrm{G}=\rho \mathrm{v}_{\mathrm{T}}^{2} \\
v=\frac{\left(\mathrm{v}_{\mathrm{L}} / \mathrm{v}_{\mathrm{T}}\right)^{2}-2}{2\left[\left(\mathrm{v}_{\mathrm{L}} / \mathrm{v}_{\mathrm{T}}\right)^{2}-1\right]}
\end{gathered}
$$

Since in addition to density in equations (2) and (3), only $\mathrm{v}_{\mathrm{L}}$ and $\mathrm{v}_{\mathrm{T}}$ (both independent of the direction of propagation in an isotropic medium) are present in equations (2) and (4), and only $\mathrm{v}_{\mathrm{T}}$ in equation (3), incidence from any one plane of an isotropic material allows for the assessment of its elastic properties.

Now, the elastic constants of a medium are directly related to the binding energy of its constituent particles, so each phase of a multiphase material possesses its own characteristic constants. Also, the presence of crystal defects has an effect on the binding energy of the particles around them, affecting the value of the constants; in fact, the interaction of elastic waves with dislocation has long been studied, so ultrasound has been used to measure dislocation density ${ }^{4}$. Thus, the measured velocity of a wave traveling through the bulk of a material will be the result of a complex interaction between 
microstructural elements like grains, grain boundaries, second phases, precipitates, pores, dislocations, interstitials, and vacancies ${ }^{5,6}$. It is expected then, that any change either in the nature or amount of the phases of a given material will result in a change in the average elastic constants and consequently in the measured velocities, as observed for microstructural changes due to tempering in alloy steels (AISI 304, 304L, 316L an 38NiCrMo 4$)^{7}$.

Based on the above, the objective of this work is to assess if ultrasound can be used to evaluate heat treatments on carbon and low alloy steels; that is, if the microstructural modifications associated to various heat treatments generate changes in the elastic constants that can be effectively measured by means of nondestructive ultrasonic techniques.

\section{Experimental Procedure}

Thirteen samples were cut from a $1 / 2$ " in diameter SAE 1045 carbon steel bar and thirteen from a $3 / 4$ " in diameter SAE 4140 low alloy steel bar. The chemical composition of both steels is shown in Table 1. The samples were all cut perpendicularly to the length of the bars to nominal thicknesses of $28 \mathrm{~mm}$, which were measured with a micrometer; then, times of flight (TOF) of ultrasonic waves were recorded. For each one of the steels, one of the samples was left as provided for reference, while the other twelve were heat treated as detailed in Table 2. A Herrmann-Moritz electric furnace was used for austenizing the samples during 30 minutes under reducing atmosphere, six at $900{ }^{\circ} \mathrm{C}$ and the other six at $1000^{\circ} \mathrm{C}$. Once austenization at each temperature was complete, four samples were quenched in water, one normalized by cooling in quiet air, and one left to cool in the furnace for annealing. Finally, three of the quenched samples were tempered in a salt bath at the temperatures and time shown in Table 2.

After the heat treatments, the samples were cut in approximate halves, that is, to $14 \mathrm{~mm}$ nominal thicknesses; their surfaces were cleaned from any rust and their thicknesses were measured with a micrometer. Then, the echo signals of longitudinal and shear waves traveling along the thickness of each half were stored. To ensure both waves traveled the same distance, the corresponding transducers were always placed on the center of the samples. Figure 1 shows the ultrasonic measurement setup. The ultrasonic system consisted of a Panametrics 5077PR pulser-receiver, on pulse-echo mode, a TiePie Scope HS805 oscilloscope (set at a sampling rate of $1 \mathrm{GHz}$ ), and two Panametrics direct-contact transducers, one for longitudinal and one for shear waves. Both transducers were $11 \mathrm{~mm}$ in diameter and of 5-MHz central frequency.

Table 1. Chemical composition of SAE 1045 and SAE 4140 steels.

\begin{tabular}{lcccccccccccc}
\hline $\begin{array}{l}\text { Element } \\
\text { (wt \%) }\end{array}$ & $\mathrm{C}$ & $\mathrm{Si}$ & $\mathrm{Mn}$ & $\mathrm{P}$ & $\mathrm{S}$ & $\mathrm{Cr}$ & $\mathrm{Ni}$ & $\mathrm{Mo}$ & $\mathrm{Al}$ & $\mathrm{Cu}$ & $\mathrm{Co}$ & $\mathrm{Ti}$ \\
\hline SAE 1045 & 0,427 & 0,154 & 0,76 & 0,029 & 0,042 & 0,128 & 0,126 & 0,013 & 0,0008 & 0,435 & 0,0068 & 0,0007 \\
SAE 4140 & 0,396 & 0,278 & 0,79 & 0,019 & 0,023 & 0,93 & 0,193 & 0,178 & 0,0068 & 0,22 & 0,0056 & 0,0018 \\
\hline
\end{tabular}

Table 2. Heat treatments of SAE 1045 and SAE 4140 steel samples.

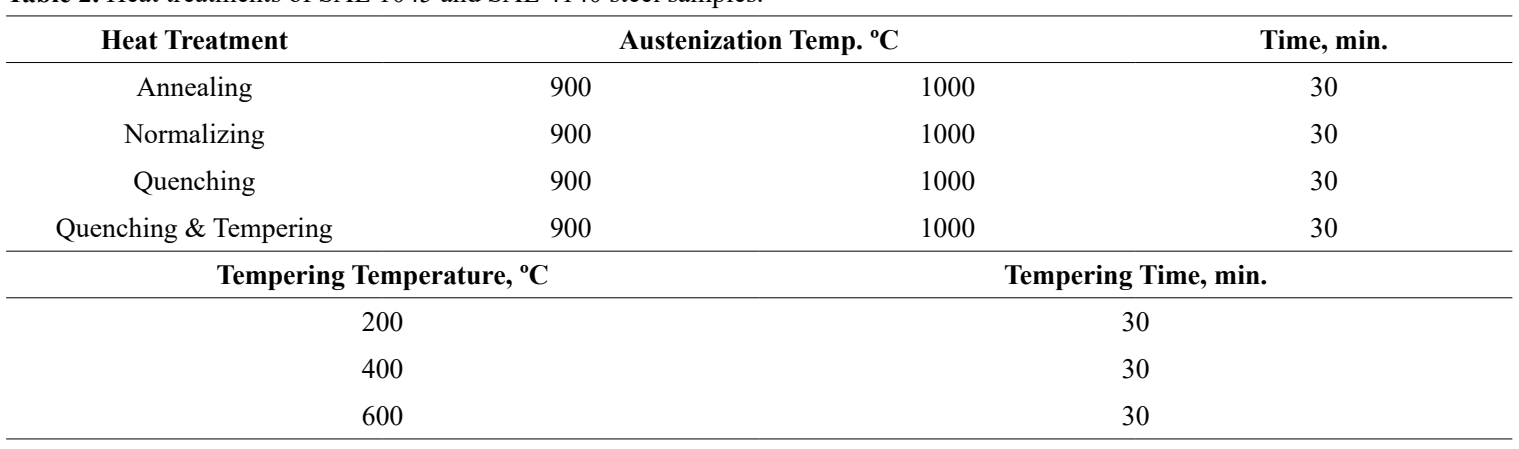

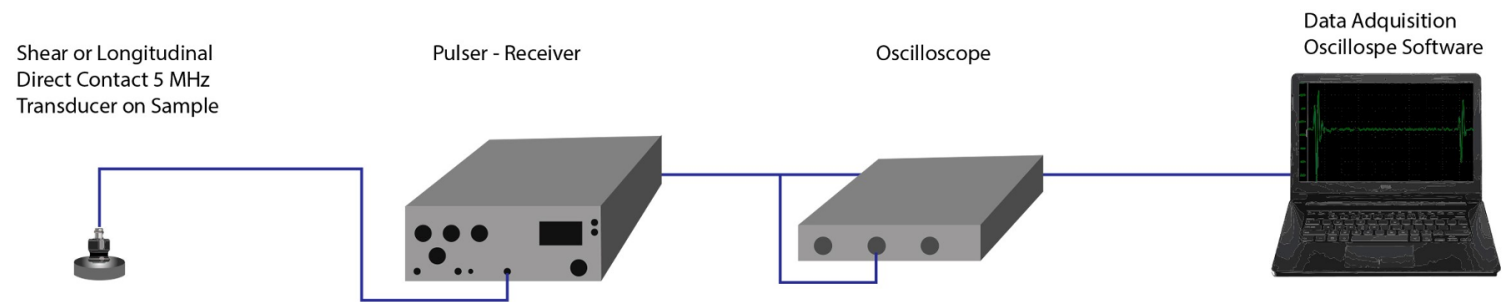

Figure 1. Ultrasonic measurement setup. 
To relate results to standard techniques, metallographic samples were prepared by common procedures, and Vickers microhardness was measured in the reference and heat treated samples. Five measures were taken on each sample for averaging.

\section{Results and Discussion}

\subsection{Microstructures}

Representative microstructures of the SAE 1045 and SAE 4140 steels that underwent different heat treatments are shown in Figures 2 and 3.
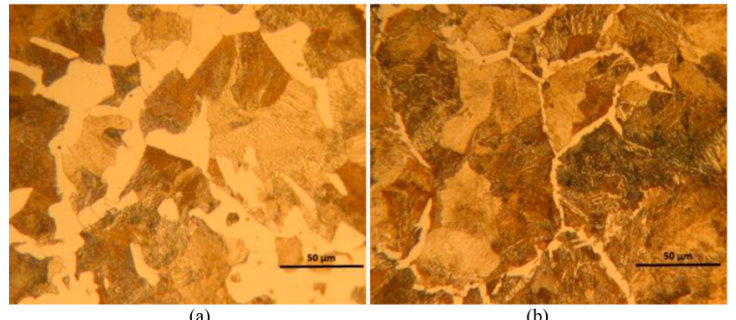

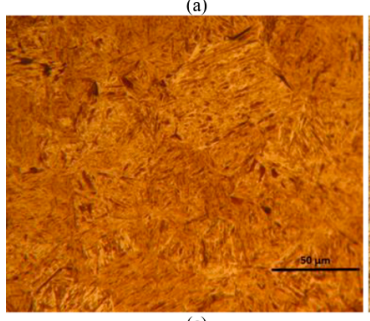

(c)

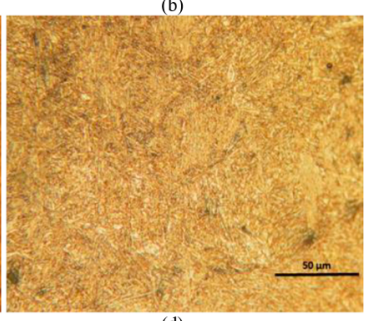

(d)
Figure 2. Microstructure of SAE 1045 steel, (a) annealed from $900{ }^{\circ} \mathrm{C}$; (b) normalized from $1000{ }^{\circ} \mathrm{C}$; (c) quenched from 1000 ${ }^{\circ} \mathrm{C}$; (d) quenched from $900{ }^{\circ} \mathrm{C}$, tempered at $400{ }^{\circ} \mathrm{C}$ for 30 minutes.

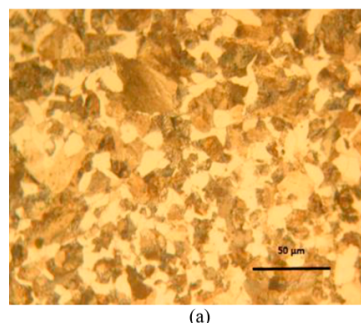

(a)

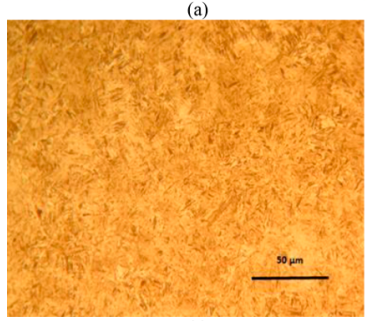

(c)

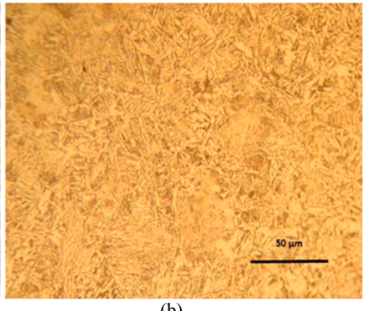

(b)

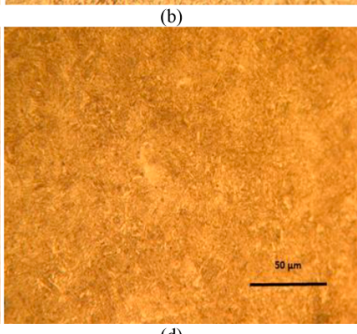

Figure 3. Microstructure of SAE 4140 steel, (a) annealed from $900{ }^{\circ} \mathrm{C}$; (b) normalized from $900{ }^{\circ} \mathrm{C}$; (c) quenched from $900{ }^{\circ} \mathrm{C}$; (d) quenched from $900{ }^{\circ} \mathrm{C}$, tempered at $400{ }^{\circ} \mathrm{C}$ for 30 minutes.
As expected, the annealed sample (Figure 2a) shows grain boundary ferrite and pearlite colonies, while the normalized one (Figure 2b) shows less primary ferrite, with presence of Widmanstätten ferrite, and fine pearlite. Meanwhile, martensite and tempered martensite are present in the quenched and quenched and tempered samples of Figures $2 \mathrm{c}$ and $2 \mathrm{~d}$.

Likewise, on the SAE 4140 steel samples, the annealing treatment produced grain boundary ferrite and pearlite colonies (Figure 3a); normalizing generated blocky and Widmanstätten ferrite, and fine pearlite colonies (Fig. 3b); and, as before, martensite and tempered martensite are present in the quenched and quenched and tempered samples shown in figures $3 \mathrm{c}$ and $3 \mathrm{~d}$.

\subsection{Hardness}

Figure 4 shows the hardness values for the heat treatments performed on both steels. As expected, they are lowest for annealing, slightly higher for normalizing and maximum for quenching. As the tempering temperature is increased from $200{ }^{\circ} \mathrm{C}$ to $600{ }^{\circ} \mathrm{C}$, hardness decreases towards the values of the normalized condition.

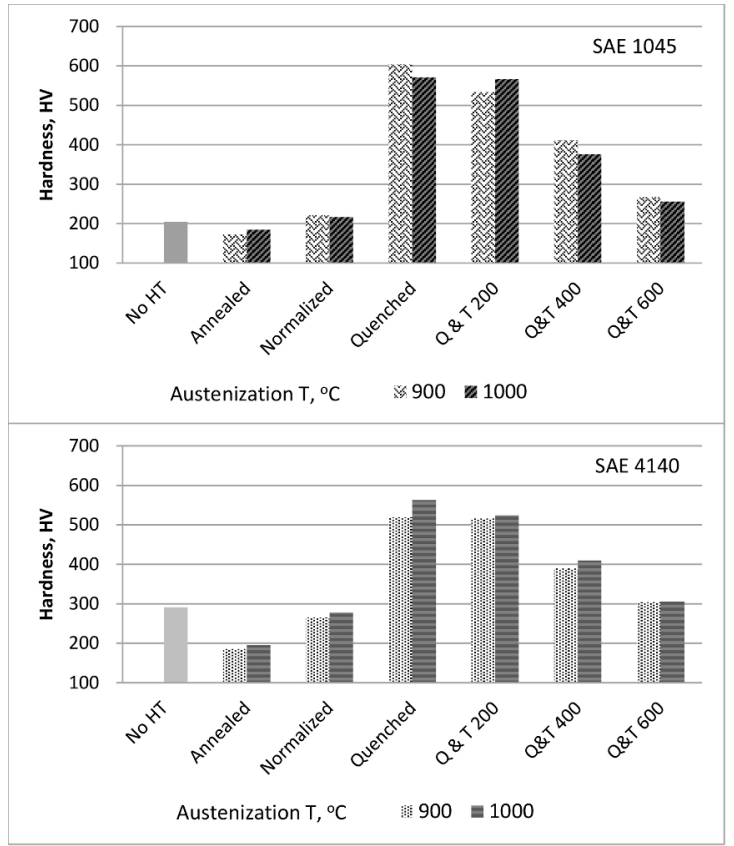

Figure 4. Hardness versus heat treatment for the SAE 1045 and SAE 4140 steels.

The graphs also show that the steels were most probably provided in a normalized state (No HT in the graphs) and that a higher austenizing temperature has a consistent but slight effect on the hardness of the low alloy steel, not so on the carbon steel. When comparing both steels, is observed that the finer microstructure of the normalized SAE 4140 steel 
has a higher hardness than the normalized structure of the SAE 1045 steel. On the contrary, the as quenched hardness is rather higher on the SAE 1045 carbon steel, probably due to the somewhat higher carbon content of its martensitic structure. Consequently, for the low and intermediate tempering temperature, the hardness of both steels is similar, while at $600{ }^{\circ} \mathrm{C}$ the 4140 steel is again harder.

\subsection{Young's and Shear Moduli}

After obtaining and storing the signals, the TOF of the waves were measured, quantified as the time difference between the highest peaks of the first two consecutive echoes (Figure 5), and the wave velocities were calculated (Table 3 ). An inspection of each column of Table 3 shows that the general trend of velocities is to decrease from annealing to quenching, and increase when tempering. This behavior is reflected on the values of the elastic constants, as explained below.

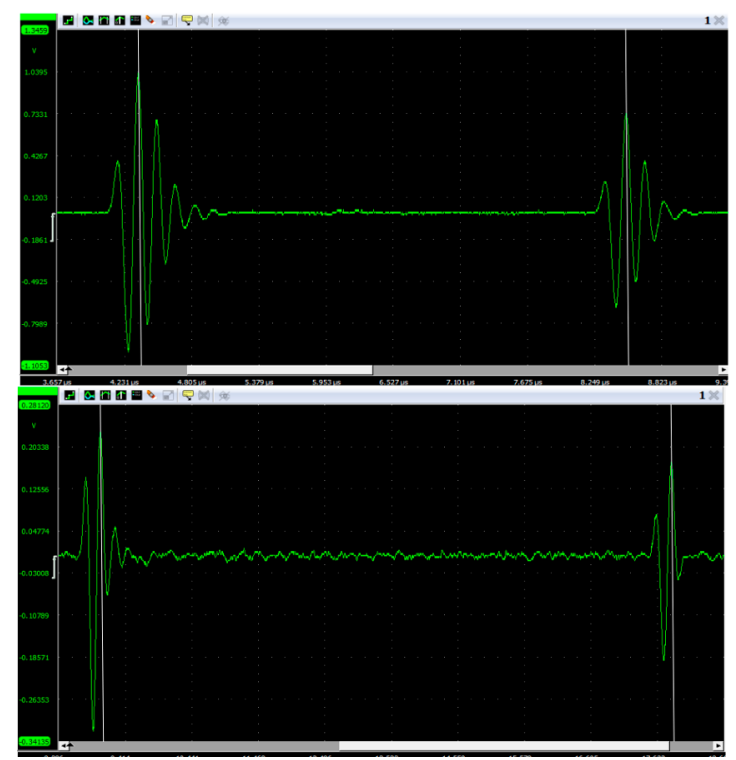

Figure 5. Echo signal of a longitudinal wave (top) and a shear wave (bottom).
To determine Young's and shear moduli, a constant density of $7800 \mathrm{~kg} \cdot \mathrm{m}^{-3}$ was assumed on equations (2) and (3). Figures 6 and 7 show these parameters as a function of the different heat treatments. The values reported at each heat treatment correspond to the average of the values obtained on both halves of the samples. On both steels the behavior is similar with the elastic moduli changing consistently with the microstructural changes; that is, E and $\mathrm{G}$ are highest in the annealed condition, decrease when normalized and reach their lowest value in the as quenched state. Also, as the tempering temperature is increased, $\mathrm{E}$ and $\mathrm{G}$ increase reaching, at $600^{\circ} \mathrm{C}$, values near those of the normalized condition. This behavior may be explained by considering the relative values of the binding energy of the constituent particles within the different phases (which in turn determine their melting or decomposition temperature) and at the interphases, and the density of dislocations and other crystal defects. Thus, the higher density of interphases in the normalized steels might help explain why E and $G$ are lower than in the annealed condition. On the other hand, martensite, which decomposes readily as temperature is raised, and has a high density of dislocations, shows the lowest values of $\mathrm{E}$ and $\mathrm{G}$. Tempering of martensite tends to produce more stable structures as the tempering temperature is raised, thus rendering higher values of $\mathrm{E}$ and $\mathrm{G}$.

Figures 6 and 7 show there is but a small, if any, effect of austenization temperature on the elastic parameters of the annealed, normalized and quenched steels. On the other hand, for the SAE 4140 steel the austenization temperature, which defines the previous austenite grain size, seems to have a consistent effect as the tempering temperature increases; however, the same is not true for the SAE 1045 steel.

It was stated that density was taken as constant for determining $\mathrm{E}$ and $\mathrm{G}$. This assumption might seem disturbing, since its actual value will depend on the microstructure of a given steel and on composition when both steels are considered. It must be realized, though, that the induced error is actually small, as can be seen if, for instance, the density

Table 3. Shear $\left(\mathrm{V}_{\mathrm{T}}\right)$ and longitudinal $\left(\mathrm{V}_{\mathrm{L}}\right)$ wave velocities versus heat treatment for the SAE 1045 and SAE 4140 steels.

\begin{tabular}{|c|c|c|c|c|c|c|c|c|}
\hline & \multicolumn{4}{|c|}{ SAE 1045} & \multicolumn{4}{|c|}{ SAE 4140} \\
\hline & \multicolumn{8}{|c|}{ Austenization Temperature ${ }^{\circ} \mathrm{C}$} \\
\hline & \multicolumn{2}{|c|}{900} & \multicolumn{2}{|c|}{1000} & \multicolumn{2}{|c|}{900} & \multicolumn{2}{|c|}{1000} \\
\hline & \multicolumn{8}{|c|}{ Wave velocity, ms $^{-1}$} \\
\hline Heat Treatment & $\mathrm{V}_{\mathrm{T}}$, & $\mathrm{V}_{\mathrm{L}}$ & $\mathrm{V}_{\mathrm{T}}$, & $\mathrm{V}_{\mathrm{L}}$ & $\mathrm{V}_{\mathrm{T}}$, & $\mathrm{V}_{\mathrm{L}}$ & $\mathrm{V}_{\mathrm{T}}$, & $\mathrm{V}_{\mathrm{L}}$ \\
\hline Annealing & 3,251 & 5,964 & 3,244 & 5,980 & 3,263 & 5,965 & 3,261 & 5,969 \\
\hline Normalizing & 3,234 & 5,934 & 3,229 & 5,907 & 3,251 & 5,954 & 3,250 & 5,952 \\
\hline Quenching & 3,185 & 5,896 & 3,186 & 5,890 & 3,204 & 5,916 & 3,207 & 5,908 \\
\hline Q \& T 200 & 3,198 & 5,911 & 3,207 & 5,913 & 3,210 & 5,927 & 3,210 & 5,915 \\
\hline Q \& T 400 & 3,219 & 5,946 & 3,221 & 5,918 & 3,218 & 5,916 & 3,226 & 5,922 \\
\hline Q \& T 600 & 3,242 & 5,927 & 3,230 & 5,946 & 3,244 & 5,942 & 3,254 & 5,946 \\
\hline No Heat Treatment & 3,224 & 5,939 & 3,224 & 5,939 & 3,247 & 5,947 & 3,247 & 5,947 \\
\hline
\end{tabular}




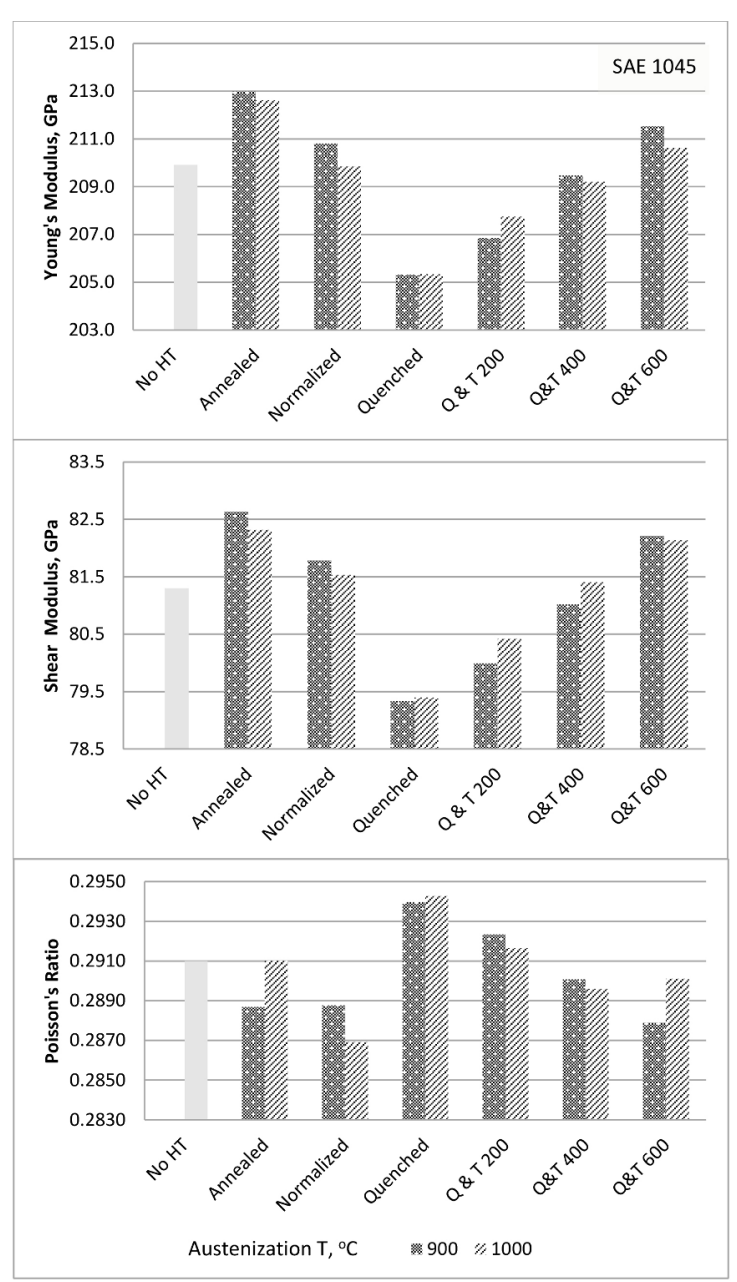

Figure 6. Elastic parameters of SAE 1045 steel as a function of heat treatment.

of martensite, approximately $7750 \mathrm{~kg} \cdot \mathrm{m}^{-3}$, was chosen for the quenched steels; $E$ and $G$ in Figures 6 and 7 would decrease even further about 1.3 and $0.5 \mathrm{GPa}$, respectively. That is, even though the exact values of the elastic constants would change if density changes were taken into account, their behavior depicted in Figures 6 and 7 would not.

Another source of error to be considered when determining $\mathrm{E}$ and $\mathrm{G}$ is the measurement of thickness. As pointed out, on this work they were carefully measured with a micrometer. If, however, a large error on the measurement of thickness is assumed, say $10 \mu \mathrm{m}$ in a $28.000 \mathrm{~mm}$ thick sample with $\mathrm{E}$ $=211.87$, the value will only shift to 212.02 , which means an error of barely $0.15 \mathrm{GPa}$, or $0.07 \%$, on the value of $\mathrm{E}$.

\subsection{Poisson's Ratio}

Figures 6 and 7 also show the behavior of Poisson's ratio. It can be observed that, in general, it behaves inversely to $\mathrm{E}$ and $\mathrm{G}$, much like hardness does (compare with Figure 4). This reflects the fact that the more rigid a medium is, the less it will contract transversely to the elongation direction.

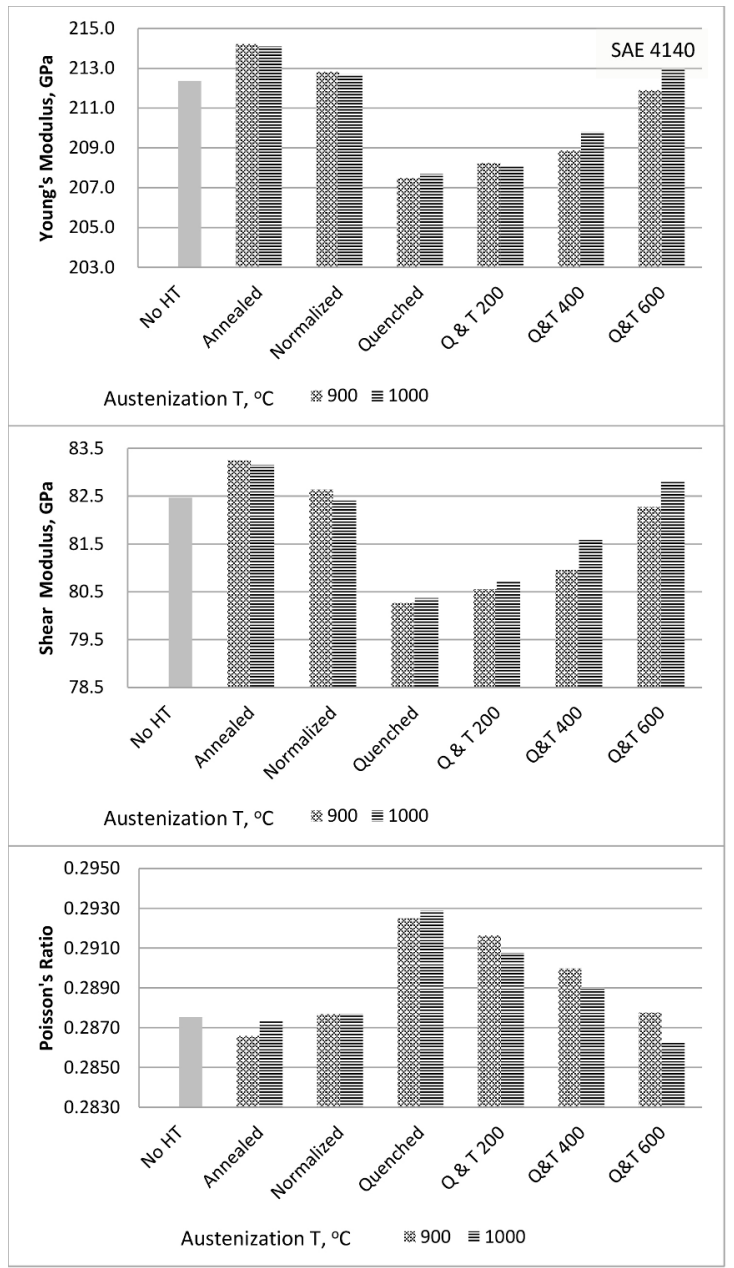

Figure 7. Elastic parameters of SAE 4140 steel as a function of heat treatment.

That is why the less rigid martensite phase has the highest value of Poisson's ratio.

Now, equation (4) shows two interesting features: first, no value of density is required and second, since only ratios of velocities enter the equation, Poisson's ratio is actually calculated as ratios of TOF of the waves traveling the same distance d. Thus, even though it was stated that under controlled conditions these sources of error were unimportant, they are not present at all when Poisson's ratio is used, and, since its behavior accompanies those of $\mathrm{E}$ and $\mathrm{G}$, this renders the previous analysis even more robust. Furthermore, in many situations, the determination of an exact value of density and a measure of thickness could be impossible, so the use of Poisson's ratio might be the choice.

In order to use this ultrasonic method to monitor heat treatments, their effects should be studied with respect to a given initial state. Figure 8 summarizes the percentage change in the elastic parameters due to the different heat treatments when the annealed state is taken as the starting point. While normalizing changes $\mathrm{E}$ and $\mathrm{G}$ in about $1 \%$, a 
change of more than $3 \%$ in $\mathrm{E}$ and up to $4 \%$ in $\mathrm{G}$ takes place due to quenching. Poisson's modulus may not be as effective to discern between the annealed and normalized states, but up to a $2 \%$ change is observed due to quenching. On the other hand, the three parameters change considerably due to tempering. This effect is enhanced when the quenched state is considered as the starting point. In fact, Figure 9 shows that as tempering temperature increases to $600{ }^{\circ} \mathrm{C}, \mathrm{E}$ and $\mathrm{G}$ increase about 3\%, while the reduction in Poisson's ratio reaches around $2 \%$.

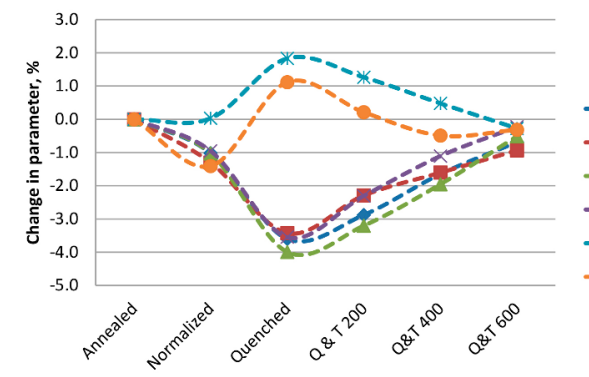

1045

$-\rightarrow-E, 900$

- - E, 1000

$-A-G, 900$

$-\not r-G, 1000$

$-*-v, 900$

$-\bullet-v, 1000$

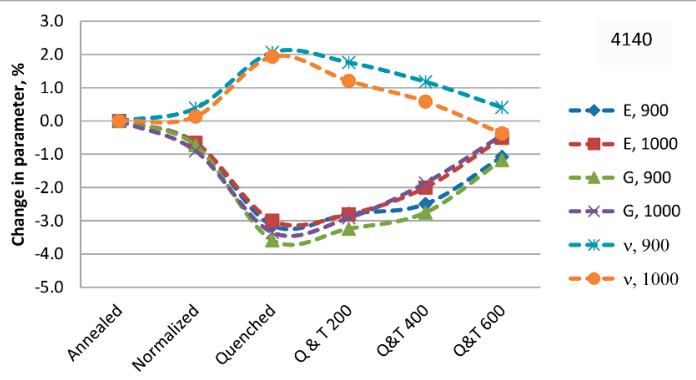

Figure 8. Change with heat treatment in elastic parameters of SAE 1045 and SAE 4140 steels, relative to the annealed state.

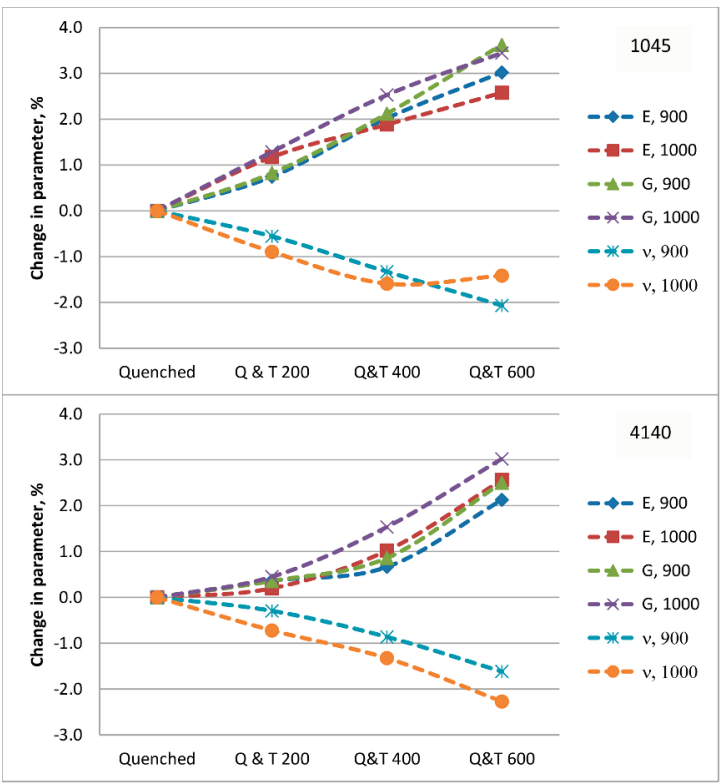

Figure 9. Change with heat treatment in elastic parameters of SAE 1045 and SAE 4140 steels, relative to the quenched state.
At this point, a final remark is necessary. In order to prove the feasibility of the method and to set it on solid bases, this study was carried on two commercial steels, taking several samples from different locations of the bars. Each one of these samples underwent a different heat treatment. Since engineering steels usually present impurities, inclusions and crystal defects that might be heterogeneously distributed, fluctuations of velocities, and densities, can be expected, and so was the case here. However, it must be realized that when putting the method to use, TOF of the ultrasonic waves will be measured at one specific location before and after the heat treatment of the part to be examined, so that such fluctuations will be absent. Also, if different locations are to be controlled, TOF will still be compared at each one.

\section{Conclusions}

It was shown experimentally that changes in elastic parameters due to different heat treatments in two common commercial steels are large enough to be discerned by measures of TOF and velocities of ultrasonic waves. Thus, ultrasound can be successfully used as a nondestructive tool to monitor heat treatments in steels by simple measures of TOF of either longitudinal or shear waves, or both.

\section{Acknowledgements}

The authors acknowledge SIMET-USACH and Proyecto Basal USA 1555 USACH-MECESUP for the financial support provided for carrying out this work.

\section{References}

1. Rose JL. Ultrasonic Waves in Solid Media New York: Cambridge University Press; 1999. p. 27-33, 272-285.

2. Dieter GE. Mechanical MetallurgyLondon: McGraw-Hill Book; 1988. p. 26-60.

3. Bray DE, Stanley RK. Nondestructive Evaluation: A Tool in Design, Manufacturing, and ServiceBoca Raton: CRC Press; 1997. p. 53-59.

4. Mujica N, Cerda MT, Espinoza R, Lisoni J, Lund F. Ultrasound as a probe of dislocation density in aluminum. Acta Materialia 2012;60(16):5828-5837.

5. Hauk V, ed. Structural and Residual Stress Analysis by Nondestructive MethodsAmsterdam: Elsevier Science; 1997.

6. Totten G, Howes M, Inoue T, eds. Handbook of Residual Stress and Deformation of SteelMaterials Park: ASM International; 2002.

7. Moro A, Farina C, Rossi F. Measurement of ultrasonic wave velocity in steel for various structures and degrees of cold working. NDT International 1980;13(4):169-175. 